\title{
Phase 1 and pre-clinical profiling of ESM-HDAC391, a myeloid-targeted histone deacetylase inhibitor, shows enhanced pharmacology and monocytopaenia
}

\author{
Rebecca Furze ${ }^{1}$, Judit Molnar ${ }^{1}$, Nigel Parr ${ }^{1}$, Faiz Ahmad ${ }^{1}$, Yvette Henry ${ }^{1}$, David Howe ${ }^{1}$, \\ Rajendra Singh ${ }^{2}$, Martin Toal ${ }^{1}$, Anna Bassil ${ }^{1}$, Sharon Bernard ${ }^{1}$, Robert Davis ${ }^{1}$, Adele \\ Gibson $^{1}$, Claire Maller ${ }^{1}$, Catriona Sharp ${ }^{1}$, David Tough ${ }^{1}$, Rab Prinjha ${ }^{1}$, and Huw Lewis ${ }^{1}$ \\ ${ }^{1}$ GlaxoSmithKline Research and Development \\ ${ }^{2}$ GlaxoSmithKline USA
}

January 18, 2022

\begin{abstract}
AIM: To improve the tolerability and therapeutic application of histone deacetylase inhibitors (HDACi), by application of an esterase-sensitive motif (ESM), to target pharmacological activity directly to mononuclear myeloid cells expressing the processing enzyme carboxylesterase-1 (CES1). METHODS. This first-in-human study comprised of single and multiple ascending dose cohorts to determine safety and tolerability. Pharmacodynamic parameters included acetylation, cytokine inhibition and intracellular concentrations of processed acid metabolite in isolated monocytes. Mechanistic work was conducted in vitro and in a CES1/Es1e lo mouse strain. RESULTS. ESM-HDAC391 was well tolerated whilst showing robust targeted mechanistic engagement, as demonstrated by selective retention of compound and increased acetylation in monocytes plus inhibition of ex vivo stimulated cytokine production. Importantly, common clinical HDACi toxicities were not observed. ESM-HDAC391 treatment was accompanied by the novel finding of a dose-dependent monocyte depletion that was transient and reversible. In-depth characterisation of monocyte depletion in a transgenic mouse model $\left(C E S 1 / E s 1 e^{l o}\right)$ suggested that CSF1R loss on circulating cells contributes to ESM-HDAC-mediated depletion. Further mechanistic investigations using human monocytes in vitro demonstrated HDACi-mediated change in myeloid fate through modulation of CSF1R and downstream effects on cell differentiation. CONCLUSION. These findings demonstrate selective targeting of monocytes in humans using the ESM approach and identify monocytopaenia as a novel outcome of ESM-HDACi treatment, with implications for the potential benefit of these molecules in myeloid-driven diseases.
\end{abstract}

\section{Hosted file}

Furze et al Manuscript BJCP_FINAL.docx available at https://authorea.com/users/456022/ articles/553223-phase-1-and-pre-clinical-profiling-of-esm-hdac391-a-myeloid-targetedhistone-deacetylase-inhibitor-shows-enhanced-pharmacology-and-monocytopaenia 